\title{
Aspirin versus placebo in pregnancies at high risk for preterm pre-eclampsia
}

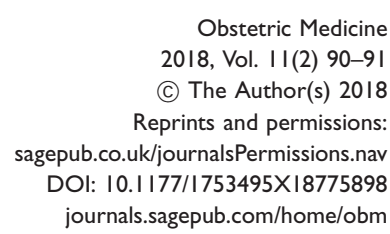

@AGE

\section{Lisa Story $\mathbb{D}$ and Catherine Nelson-Piercy}

\begin{abstract}
A recent multicentre placebo-controlled trial by Rolnick et al. has indicated that taking $150 \mathrm{mg}$ aspirin daily in women at high risk of pre-eclampsia reduced the incidence of the disease from $4.3 \%$ to $1.5 \%$ in comparison with placebo. Although the findings of this study are important, a high proportion of women withdrew their consent and not all safety outcomes have been reported. This journal watch article discusses the paper in more detail.
\end{abstract}

\section{Keywords}

Aspirin, pre-eclampsia

Received 30 November 2017; accepted I6 April 2018

A recent multicentre placebo-controlled trial by Rolnick et al. has indicated that taking $150 \mathrm{mg}$ aspirin nocte in women at high risk of pre-eclampsia reduced the prevalence of preterm pre-eclampsia in comparison with placebo from 4.3 to $1.5 \%{ }^{1}$ This journal watch article discusses the paper in more detail as well as a separate subgroup analysis of the data, published by Poon et al., specifically assessing the benefit in certain patient groups. ${ }^{2}$

Pre-eclampsia is a pregnancy-specific multisystem condition, associated with significant morbidity for both the mother and fetus Delivery is currently the only definitive treatment; however, previous studies have indicated that its incidence can be reduced by $10 \%{ }^{3}-50 \%{ }^{4,5}$ by administration of prophylactic low-dose aspirin early in pregnancy to high-risk women. The maximum benefit of aspirin has previously been found to be conferred when commenced prior to 16 weeks' gestation. ${ }^{4,5}$

Rolnick et al. conducted a multicentre double-blind placebocontrolled trial which randomised, 1776 women with singleton pregnancies at high risk of pre-eclampsia to receive $150 \mathrm{mg}$ aspirin daily or placebo every night from 11 to 14 weeks until 36 weeks' gestation. Patients were deemed to be at high risk based on an algorithm combining: maternal factors, mean arterial pressure, uterine artery pulsatility index, serum pregnancy-associated plasma protein A and placental growth factor. The primary outcome was pre-eclampsia culminating in delivery before 37 weeks' gestation. Treatment with aspirin was found to significantly reduce the incidence of preterm preeclampsia from $4.3 \%$ in the placebo group to $1.5 \%$ in the treatment group (odds ratio in the aspirin group $0.38 ; 95 \%$ confidence interval, 0.2 to $0.74 ; \mathrm{P}=0.004)$. No effect on the incidence of term preeclampsia was noted.

In addition, the group published a second paper assessing whether there were differences in the effect of aspirin on the incidence of preterm pre-eclampsia in certain subgroups: maternal age $<30$ and $\geq 30$, BMI $<25$ and $\geq 25$, racial origin, method of conception, smoking, family history of preterm pre-eclampsia, obstetric history, and history of chronic hypertension (present and past). ${ }^{2}$ They reported aspirin to be beneficial in all subgroups with the exception of chronic hypertension. In the chronic hypertensive cohort, preterm pre- eclampsia occurred in $10.2 \%(5 / 49)$ in the aspirin group and $8.2 \%$ $(5 / 61)$ in the placebo group (adjusted odds ratio $1.29,95 \%$ confidence interval 0.33-5.12). In those without chronic hypertension, preterm pre-eclampsia occurred in $1.1 \%(8 / 79)$ in the aspirin group and $3.9 \%$ $(30 / 761)$ in the placebo group (adjusted odds ratio, 0.27 ; $95 \%$ confidence interval, 0.12-0.60). However, the statistical power for detecting effects in these smaller subgroups was poor.

Although these findings are significant, it should be noted that $8.6 \%$ of women, withdrew their consent during the course of the study and were excluded from the analyses. The reasons for this are not discussed but may be related to the fact that aspirin had previously been shown to reduce the incidence of pre-eclampsia and if eligible women elected to participate there was a $50 \%$ chance they may have been deprived of this benefit. This may be particularly relevant as aspirin is freely available as an over-the-counter medication which women could purchase independently. Although the authors state that a sensitivity analysis performed to evaluate the effect of the withdrawals showed no substantive difference from the primary analysis, of the 152 women who withdrew consent, 74 did not want any of their data to be reported. The impact on the validity of the sensitivity analysis should therefore be questioned.

A dose of $150 \mathrm{mg}$ of aspirin was used in this trial which is in contrast to the current NICE guidance $(75 \mathrm{mg})^{6}$ and ACOG recommendations $(60-80 \mathrm{mg}) .^{7}$ Recent work by Roberge et al. has indicated a dose-response relationship up to $150 \mathrm{mg}$ of aspirin in the prevention of early onset pre-eclampsia; ${ }^{8}$ however, the incidence of adverse outcomes, which may be associated with higher doses of aspirin, such as gastritis has not been reported in this study. In addition, it is

Department of Women's and Children's Health, King's College London, St Thomas' Hospital, London, UK

\section{Corresponding author:}

Catherine Nelson-Piercy, St Thomas' Hospital, London SEI 7EH, UK.

Email: catherine.nelson-piercy@gstt.nhs.uk 
unknown whether the dosing should be altered in relation to patient's body mass index. Studies in non-pregnant participants have indicated that platelet responsiveness is reduced in obese patients. Although the subgroup analysis by Poon et al. found no effect of BMI on the effectiveness of aspirin, only two groups were analysed (BMI $<25$ and $\mathrm{BMI} \geq 25)$ and numbers were small $(5 / 311$ women who took aspirin developed pre-eclampsia as opposed to $14 / 338$ who took placebo in women who had a BMI $<25 \mathrm{~kg} / \mathrm{m}^{2}$, and $8 / 487$ women who took aspirin developed pre-eclampsia as opposed to $21 / 484$ women who took the placebo who had a BMI $\geq 25 \mathrm{~kg} / \mathrm{m}^{2}$ ). Further studies are therefore required to allay concerns regarding, optimal dosage ( $75 \mathrm{mg}$ versus $150 \mathrm{mg}$ ), efficacy in different population groups, perinatal and other safety outcomes.

Regarding translation into clinical practice, the identification of patients at high risk of pre-eclampsia in this study was done by means of an algorithm comprising both serum and ultrasonographic predictors, not all of which are currently used in routine clinical practice, such as placental growth factor, and the timings of investigations in current clinical practice are also different (for example, uterine artery Doppler Pulsatility Index is not routinely performed at 11-13 weeks' gestation in most units). The cost implications of undertaking these additional measurements also need to be considered and a cost benefit analysis should be performed before it is introduced into clinical practice.

The findings of this paper, that treatment with $150 \mathrm{mg}$ nocte of aspirin in high-risk women results in a lower incidence of preterm pre-eclampsia than placebo, and the performance of this predictive/ preventative model can be reproduced, are likely to have a significant impact on both maternal and perinatal outcomes.

\section{Declaration of conflicting interests}

The author(s) declared no potential conflicts of interest with respect to the research, authorship, and/or publication of this article.

\section{Funding}

The author(s) received no financial support for the research, authorship, and/or publication of this article.

\section{Guarantor}

CN-P guarantees the manuscript's accuracy and the contribution of all authors.

\section{Contributorship}

LS wrote the first draft. Both authors edited first, subsequent and final versions.

\section{ORCID iD}

Lisa Story (D) http://orcid.org/0000-0001-9328-9592

\section{References}

1. Rolnik DL, Wright D, Poon LC, et al. Aspirin versus placebo in pregnancies at high risk for preterm preeclampsia. $N$ Engl J Med 2017; 377: 613-622.

2. Poon LC, Wright D, Rolnik DL, et al. Aspirin for evidence-based preeclampsia prevention trial: effect of aspirin in prevention of preterm preeclampsia in subgroups of women according to their characteristics and medical and obstetrical history. Am J Obstet Gynecol 2017; 217: 585.e1-585.e5.

3. Askie LM, Duley L, Henderson-Smart DJ, et al. Antiplatelet agents for prevention of pre-eclampsia: a meta-analysis of individual patient data. Lancet 2007; 369: 1791-1798.

4. Bujold E, Roberge S, Lacasse Y, et al. Prevention of preeclampsia and intrauterine growth restriction with aspirin started in early pregnancy: a meta-analysis. Obstet Gynecol 2010; 116: 402-414.

5. Roberge S, Nicolaides KH, Demers S, et al. Prevention of perinatal death and adverse perinatal outcome using low-dose aspirin: a meta-analysis. Ultrasound Obstet Gynecol 2013; 41: 491-499.

6. NICE. Hypertension in pregnancy: diagnosis and management. London: NICE, 2010.

7. American College of Obstetricians and Gynecologists and Task Force on Hypertension in Pregnancy. Hypertension in pregnancy. Report of the American College of Obstetricians and Gynecologists' Task Force on Hypertension in Pregnancy. Obstet Gynecol 2013; 122: 1122-1131.

8. Roberge S, Nicolaides K, Demers S, et al. The role of aspirin dose on the prevention of preeclampsia and fetal growth restriction: systematic review and meta-analysis. Am J Obstet Gynecol 2017; 216: 110-120.e6. 